\title{
An Analytic Motion Planning Solution for the Snakeboard.
}

\author{
Elie Shammas \\ Department of Mechanical Engineering \\ American University of Beirut \\ Beirut, Lebanon \\ Email: es34@aub.edu.lb
}

\author{
Maurício de Oliveira \\ Department of Mechanical and Aerospace Engineering \\ University of California San Diego \\ 9500 Gilman Dr., La Jolla, CA 92093, USA \\ Email: mauricio@ucsd.edu
}

\begin{abstract}
This paper provides a closed-form analytical solution to the motion planning problem for the Snakeboard. Given a desired planar trajectory in the fiber space, an explicit solution is computed for the gaits in the base space that locomote the Snakeboard along the desired trajectory. This is achieved by introducing a new momentum-like variable that simplifies the Snakeboard's equations of motion to allow for such an explicit gait generation technique.
\end{abstract}

\section{INTRODUCTION}

The Snakeboard was first analyzed as a simple nonholonomic mechanical system in Ostrowski et al. [5]. Since then the Snakeboard has been used as an excellent platform to demonstrate important results that range from the motion planning of underactuated systems to the controllability of nonholonomic systems. The Snakeboard is in fact a simple yet nontrivial system to experiment with motion planning and controllability without being overwhelmed by complex expressions. Nevertheless, its non-trivial dynamics have so far defied efforts to provide a closed-form analytical gait generation techniques to solve its motion planning problem. The main contribution of the present paper is a solution to this problem.

The early motion planning work for the Snakeboard in Ostrowski et al. [5] used sinusoidal steering techniques first developed in Murray and Sastry [4]. This technique identified the frequencies of the sinusoidal inputs to locomote the Snakeboard along three primitive directions: forward motion, parallel parking, and rotation. In this method, the amplitudes of the input sinusoids were empirically computed. Later Ostrowski et al. [6] extended this approach to perform optimal gait analysis.

The motion planning problem for the Snakeboard was also attempted by Shammas et al. [10]. Their technique intuitively analyzed the geometric and dynamic phase shifts in order to propose gaits that locomote the Snakeboard along the desired direction. However, the analysis of the phase shifts was not automated and did not address the motion planning problem of driving the Snakeboard from an initial to a goal configuration.

An analytical solution to the motion planning problem for the Snakeboard was presented in Bullo and Lewis [1] where the problem of driving the Snakeboard from an initial to a goal state was addressed. Their body of work built upon the decoupling vector field analysis developed by Bullo and Lynch [2]. In fact Bullo and Lewis [1] addressed the motion planning problem by solving the nonlinear inversion problem via an ad hoc concatenation of allowable trajectories between the start and end fiber configuration. These kinematic trajectories ensured that the Snakeboard had zero initial and final velocity at the start and end configurations.

Where as Bullo and Lewis [1] limited themselves to kinematic trajectories, no such limitation is used in this paper. Moreover, the start and end configurations were the input to the motion planning problem in Bullo and Lewis [1], in this paper the input is an entire trajectory from a start to an end fiber configuration.

In this paper, we build upon the prior body of work and present an analytical closed-form solution to the motion planning problem for the Snakeboard. In other words, we compute the gaits that locomote the Snakeboard along a desired trajectory between two configurations. This sufficiently smooth desired trajectory can be generated by other techniques to achieve important tasks such as obstacle avoidance or to optimize an interesting metric. In this paper, we analytically solve for the gaits that drive the Snakeboard exactly along a desired trajectory.

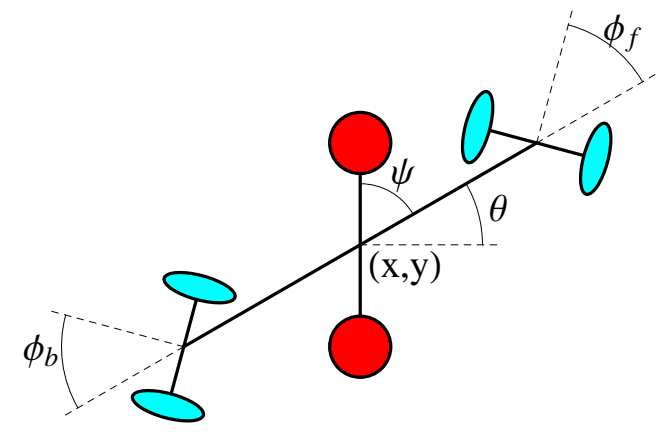

Fig. 1. Configuration variables of the Snakeboard.

This paper is organized as follows. In Section $\amalg$ we formulate the standard and reduced equations of motion for the Snakeboard. Then in Section [II] a new momentum-like variable is introduced to simplify the equations of motion so that they can be used to explicitly generate gaits that locomote 
the Snakeboard to traverse a desired trajectory. In Section IV several examples are worked out to generate gaits for specific trajectories. Finally, we discuss some of the findings of this paper in Section V and conclude in Section VI

\section{EQUATIONS OF MOTION}

The Snakeboard is a multi-bodied mechanical system with two nonholonomic constraints acting on its wheel sets. We use the Lagrangian approach to compute the Snakeboard's equations of motion. The configuration of the Snakeboard can be represented by $q=\left\{x, y, \theta, \phi_{f}, \psi, \phi_{b}\right\}$ where $x, y$, and $\theta$ denote the position and orientation of a body attached coordinate frame while $\phi_{f}, \psi, \phi_{b}$ denote the front wheel angle, the rotor angle, and the back wheel angle, respectively. Note that these angles are measured with respect to the middle link of the Snakeboard as shown in Figure 1.

The actuated variables are the rotor rotation as well as the directions of the wheels; Moreover, we assume that the wheel directions are coupled such that $\phi_{f}=-\phi_{b}=\phi$. Note that the wheels themselves are passive and are used to provide the nonholonomic constraints that permit motion only along a direction perpendicular to the wheel axes. The center of mass of the Snakeboard is at the center of the middle link where we have attached the body-coordinate frame. The location of the center of mass remains constant with respect to the body attached coordinate frame irrespective of the configuration of the Snakeboard. The mass and inertia of Snakeboard is denoted respectively by $M$ and $J$, whereas $J_{w}$ and $J_{r}$ denote the wheel and rotor inertias. The length of the middle line is assumed to be $2 L$. Finally for simplifying the expressions appearing in the equations of motions, we assume $M L^{2}=J+J_{r}+$ $2 J_{w}$. We will show how to remove these assumptions later in Section V-A

\section{A. Euler-Lagrange equations of motion}

Utilizing standard Lagrangian dynamics formulation, the equations of motion for the Snakeboard can be computed by

$$
\frac{d}{d t}\left(\frac{\partial L(q, \dot{q})}{\dot{q}_{i}}\right)-\frac{\partial L(q, \dot{q})}{q_{i}}+\sum_{j=1}^{l} \lambda_{j} \omega_{i}^{j}(q)=\tau_{i}
$$

where $L(q, \dot{q})$ is the Snakeboard Lagrangian, $\omega_{i}^{j}(q)$ is a matrix representing the nonholonomic constraints, and $\lambda_{j}$ are the Lagrange multipliers for the $l$ nonholonomic constraints. Typically, the nonholonomic constraints are expressed as $\omega(q) \dot{q}=$ 0. For the Snakeboard, the Lagrangian and the two nonholonomic constraints are respectively given by

$$
L(q, \dot{q}):=J_{r} \dot{\psi}\left(\dot{\theta}+\frac{\dot{\psi}}{2}\right)+J_{w} \dot{\phi}^{2}+\frac{M}{2}\left(L^{2} \dot{\theta}^{2}+\dot{x}^{2}+\dot{y}^{2}\right),
$$

and

$$
\omega:=\left(\begin{array}{ccccc}
-\sin (\theta+\phi) & \cos (\theta+\phi) & L \cos (\phi) & 0 & 0 \\
-\sin (\theta-\phi) & -\cos (\theta-\phi) & L \cos (\phi) & 0 & 0
\end{array}\right) .
$$

Using the above expressions for the Lagrangian and the nonholonomic constraints, the equations of motion in (1) can be computed and simulated numerically for any given motor torque input, $\tau$. Note that, the motor input vector could be represented as $\tau=\left(0,0,0, \tau_{r}, \tau_{w}\right)^{T}$.

\section{B. Reduced equations of motion}

The configuration space of the Snakeboard is a principal fiber bundle, where the fiber space is the Special Euclidean group, $g=(x, y, \theta) \in G=S E(2)$, and the base space is composed of two rotation groups, $r=(\phi, \psi) \in M=\mathbb{S}^{1} \times \mathbb{S}^{1}$, such that, $q=(g, r) \in Q=(G, M)$. The above equations of motion can be recomputed using the symmetry laws and the nonholonomic connection as defined in Ostrowski [7]. A new reduced set of equations that govern the dynamics of locomotion are given by

$$
\begin{aligned}
& \xi=-A(r) \dot{r}+\Gamma(r) p, \text { and } \\
& \dot{p}=\frac{1}{2}(p, \dot{r}) \sigma(r)(p, \dot{r})^{T},
\end{aligned}
$$

where in (2) we have $\xi=T_{g} L_{g^{-1}} \dot{g}$ is the body velocity and $A(r)$ is the local form of the mixed-nonholonomic connection and (3) is the momentum evolution equation.

In Shammas et al. [8] the above equations of motion were further simplified by introducing the scaled momentum variable $\rho(t):=f(r) p(t)$, where $f(r)$ is the integrating factor of (3), obtaining

$$
\begin{aligned}
& \xi=-A(r) \dot{r}+\frac{1}{f(r)} \Gamma(r) \rho, \text { and } \\
& \dot{\rho}=\frac{1}{2} \dot{r} \Sigma(r) \dot{r}^{T},
\end{aligned}
$$

where (5) is the scaled momentum evolution equation. Note that the right hand side of (5) is independent of any momentum variables. For the snakeboard, the reduced equation of motion in terms of the scaled momentum are computed as follows

$$
\begin{aligned}
\xi_{x} & =-\frac{J_{r} \sin (2 \phi)}{2 M L} \dot{\phi}+\frac{\cos (\phi)}{M L} \rho, \\
\xi_{y} & =0, \\
\xi_{\theta} & =-\frac{J_{r} \sin (\phi)^{2}}{M L^{2}} \dot{\phi}+\frac{\sin (\phi)}{M L^{2}} \rho, \text { and } \\
\dot{\rho} & =J_{r} \cos (\phi) \dot{\phi} \dot{\psi} .
\end{aligned}
$$

The definition of the scaled momentum, $\rho$, and the above reduced equations of motion are at the core of the motion planning techniques developed in Shammas et al. [10]. In fact, the local form of the connection in (4) was used to define height functions under which the volume was related to the geometric phase shift and the $\Gamma$ functions along with the reduced momentum equation in (4) and (5) simplified the analysis of the dynamic phase shift. The intuitive analysis of both the geometric phase shift in [9] and the dynamic phase shift in [10] allowed to propose gaits that guarantee motion along one fiber direction. In this paper we build upon the above formulation and present an explicit analytic gait generation technique for the Snakeboard. 


\section{Alternative derivation of the reduced equations of motion}

In the previous section, the symmetry of the Lagrangian and nonholonomic constraints with respect to rigid body transformation in the fiber space were exploited to recompute the equations of motion in their reduced form as shown in (2) and (3); however, in the case of the Snakeboard, this reduction step is not necessary to obtain these equations.

In fact, the equations in (2) can be computed in the inertial frame as follows. First, express the nonholonomic equations

$$
\omega_{\xi}(q) \dot{g}+\omega_{r}(q) \dot{r}=0,
$$

where $\omega_{\xi}(q)$ and $\omega_{r}(q)$ are sub-matrices of $\omega(q)$. Then define momentum variables from the derivative of the Lagrangian with respect to the fiber velocities which are then projected along the allowable directions to arrive at

$$
p_{i}=\sum_{j} \frac{\partial L(q, \dot{q})}{\partial \dot{g}_{i}} \Omega_{i}^{j}(q),
$$

where $\Omega(q)$ is a basis of the null space of $\omega_{\xi}(q)$ representing the allowable direction of motion orthogonal to the nonholonomic constraints. Solving the above equations for the fiber velocities, $\dot{g}$, will yield the reconstruction equations which are expressed in the inertial frame. The fiber velocity can then be mapped to the body frame using the lifted action of the fiber space, $\dot{q}=T_{e} L_{g} \xi$, to arrive at the equations (6) through (8).

As for the momentum evolution equations given in (3), they can be computed by using the reconstrunction equations and their derivatives to substitute for the fiber velocities, $\dot{g}$, and acceleration, $\ddot{g}$, in the original equations of motion (1D associated with the fiber variables. In turn, these equation can be solved for the derivative of the momentum variables as well as for the Lagrange multipliers. For the Snakeboard, we have two nonholonomic constraints and the fiber space, $S E(2)$, is three-dimensional; hence, only one momentum variable is required as shown in (9).

\section{EXPLICIT GAIT GENERATION}

In this section, we solve for the exact gait that will locomote the Snakeboard along a desired trajectory in the fiber space.

First introduce the new variable

$$
\delta=\rho-J_{r} \sin (\phi) \dot{\psi}
$$

so that

$$
\dot{\delta}=-J_{r} \sin (\phi) \ddot{\psi}
$$

where we have used (9) to arrive at the equivalent equations of motion

$$
\begin{aligned}
\dot{x} & =\frac{\cos (\theta) \cos (\phi)}{M L} \delta, \\
\dot{y} & =\frac{\sin (\theta) \cos (\phi)}{M L} \delta, \\
\dot{\theta} & =\frac{\sin (\phi)}{M L^{2}} \delta, \\
\dot{\delta} & =-J_{r} \sin (\phi) \ddot{\psi} .
\end{aligned}
$$

Note what we have reverted back the inertial frame rather then the body frame using $T_{e} L_{g} \xi=\dot{g}$. The main advantage of the above formulation is that it enables one to solve for $\psi$ and $\phi$ in order to force the trajectory of the Snakeboard to follow a given feasible trajectory, as discussed in the next section.

\section{A. Solution in terms of a given trajectory}

We now show how one can explicitly solve for the gait that generates a given curve in the fiber space. In other words, given a planar trajectory $c(t)=(\tilde{x}(t), \tilde{y}(t))$ which is sufficiently smooth 1 , we show how to solve equations (12) through (15) for the base variables $(\phi(t), \psi(t))$ so that $x(t) \equiv \tilde{x}(t)$ and $y(t) \equiv \tilde{y}(t)$. Note that, in general, this may not be possible, as we have to match four coupled differential equations (12) through (15) with the help of only two parameters.

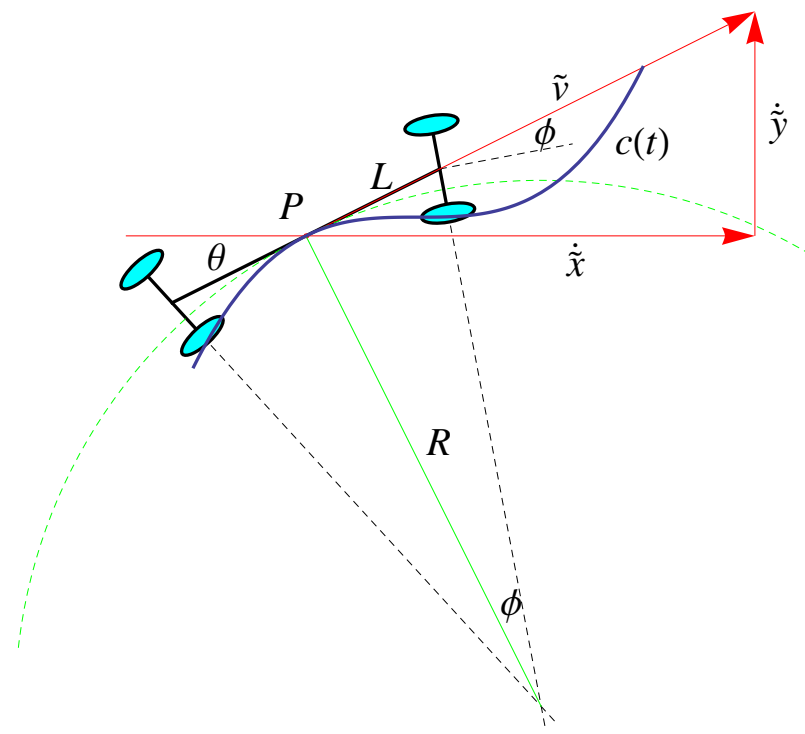

Fig. 2. Relationship between the trajectory's curvature and the wheel direction angle, $\phi$.

The first clue is to note that the vector $(\dot{x}, \dot{y})$ makes an angle $\theta$ with the horizontal. Let $\varphi(t)$ be the angle that the tangent to the trajectory makes with the horizontal inertial axis. Then 2

$$
\varphi:=\tan ^{-1}(\dot{y}, \dot{x})
$$

Dividing (13) by (12) we note that

$$
\varphi=\theta \text {. }
$$

That is, the angle that the middle link of the Snakeboard and the angle that the tangent vector $(\dot{x}, \dot{y})$ make with the horizontal are exactly the same. If we are to have $x(t) \equiv \tilde{x}(t)$ and $y(t) \equiv \tilde{y}(t)$ then we must have

$$
\varphi=\theta=\tilde{\theta}:=\tan ^{-1}(\dot{\tilde{y}}, \dot{\tilde{x}}) .
$$

\footnotetext{
${ }^{1}$ In this paper, sufficiently smooth functions are functions that are twice differentiable and their second derivatives are continuous.

${ }^{2}$ Here $\tan ^{-1}: \mathbb{R} \times \mathbb{R} \rightarrow(-\pi, \pi]$ denotes an extended version of the $\tan ^{-1}$ that uses the knowledge of the signs of $\dot{y}, \dot{x}$ in order to map the entire unit circle.
} 
The above equation completely determines $\theta(t)$.

Now think of the momentum $\delta$ as a free variable for the time being. With $x(t) \equiv \tilde{x}(t), y(t) \equiv \tilde{y}(t)$, and $\theta(t) \equiv \tilde{\theta}(t)$, consider now the question of whether it is possible to determine $(\phi, \delta)$ such that

$$
\begin{aligned}
\dot{\tilde{x}} & =\frac{\cos (\tilde{\theta}) \cos (\phi)}{M L} \delta, \\
\dot{\tilde{y}} & =\frac{\sin (\tilde{\theta}) \cos (\phi)}{M L} \delta, \\
\dot{\tilde{\theta}} & =\frac{\sin (\phi)}{M L^{2}} \delta .
\end{aligned}
$$

In order to answer this question define $\tilde{u}(t)$ and $\tilde{v}(t)$ as follows

$$
\tilde{u}:=\dot{\tilde{x}} \ddot{\tilde{y}}-\ddot{\tilde{x}} \dot{\tilde{y}}, \quad \tilde{v}:=\sqrt{\dot{\tilde{x}}^{2}+\dot{\tilde{y}}^{2}} \geq 0 .
$$

Having solved for $\tilde{\theta}$ we can compute its time derivative as well as its sine and cosine

$$
\dot{\tilde{\theta}}=-\tilde{u} / \tilde{v}^{2}, \quad \cos (\tilde{\theta})=\dot{\tilde{x}} / \tilde{v}, \quad \sin (\tilde{\theta})=\dot{\tilde{y}} / \tilde{v} .
$$

The above relationships are graphically illustrated in Figure 2

Assume now that $\tilde{v}>0$. Then either $\dot{\tilde{x}}$ or $\dot{\tilde{y}}$ or both are not zero. For instance, assume that $\dot{\tilde{x}} \neq 0$. Then we can solve for the wheel angle $\phi$ by dividing (19) by (17). After some algebra one obtains

$$
\phi=\tilde{\phi}:=-\tan ^{-1}\left(L \tilde{u} / \tilde{v}^{3}\right) .
$$

Otherwise, if $\dot{\tilde{x}}=0$ and $\dot{\tilde{y}} \neq 0$, one can now solve for $\phi$ by dividing (19) by (18). Remarkably, one reaches the exact same expression for $\phi$. Indeed, this result can be interpreted again by referring to Figure 2, where the tangent to the curve is turning clockwise as it passes through the point $P$. In this case the curvature of the curve is negative, $\kappa<0$, and we can conclude that the radius of curvature is given by $R=-1 / \kappa$. In other words

$$
\tan \phi=\frac{L}{R}=-L \kappa=-L \frac{\tilde{u}}{\tilde{v}^{3}}=\tan \tilde{\phi},
$$

where we have used that fact that for a planar curve we have

$$
\kappa=\frac{\dot{x} \ddot{y}-\dot{y} \ddot{x}}{\left(\dot{x}^{2}+\dot{y}^{2}\right)^{3 / 2}}=\frac{\tilde{u}}{\tilde{v}^{3}} .
$$

We shall also compute the sine and cosine of $\tilde{\phi}$ which will be used later

$$
\sin (\tilde{\phi})=-\frac{L \tilde{u}}{\sqrt{L^{2} \tilde{u}^{2}+\tilde{v}^{6}}}, \quad \cos (\tilde{\phi})=\frac{\tilde{v}^{3}}{\sqrt{L^{2} \tilde{u}^{2}+\tilde{v}^{6}}} .
$$

It is interesting to note that for the Snakeboard traversing a planar trajectory, the tangent of angle that the wheels make with the middle link is exactly the half the length of the middle link multiplied by the trajectories curvature.

Using the assumption that $\tilde{v}>0$ we solve for $\delta$ using (19) where we have already computed $\dot{\tilde{\theta}}$ and $\sin (\tilde{\phi})$ to arrive at

$$
\delta=\tilde{\delta}:=\frac{M L \sqrt{\tilde{v}^{6}+L^{2} \tilde{u}^{2}}}{\tilde{v}^{2}} .
$$

Finally, we can solve for the second time derivative of the rotor angle using (15). After some more algebraic manipulations we arrive at the second order differential equation

$$
\ddot{\psi}=\frac{L^{2} M \dot{\tilde{u}}}{\tilde{v}^{2} J_{r}}-\frac{2 L^{2} M \tilde{u} \dot{\tilde{v}}}{\tilde{v}^{3} J_{r}}+\frac{M \tilde{v}^{3} \dot{\tilde{v}}}{\tilde{u} J_{r}} .
$$

The solution of the above differential equation, which we shall denote $\tilde{\psi}$, can be computed explicitly. Thus, using 21 , and 24 one can compute an explicit parametrization for the wheel rotation $\tilde{\phi}$ and the rotor rotation $\tilde{\psi}$ with respect to time in terms of the desired trajectory.

\section{B. Rotor initial conditions}

To solve for $\psi$, we need to integrate (24), hence, we need the initial conditions for $\psi$, namely, $\psi(0)$ and $\dot{\psi}(0)$. The initial orientation of the rotor can be arbitrary and we can set it to zero without any loss of generality, that is, $\psi(0)=0$. As for the initial angular velocity of the rotor, we need to consider two cases.

1) Case 1: $\phi(0)=0$ : In this case, the wheel axes are perpendicular to the middle line of the Snakeboard. Hence, any initial velocity of the rotor is valid since it does not contribute to any motion. Thus, the initial velocity of the rotor can be set arbitrarily. In fact, the different initial rotor velocity will affect the momentum and scaled momentum variable and of course the rotor angle; however, it will not affect the trajectory traversed nor it will affect the $\delta$ momentum variable that was introduced in 107. This will be demonstrated later in the examples and can be seen in Figure 9 . So without any loss of generality for the case of $\phi(0)=0$, we can use the following initial conditions

$$
\psi(0)=0, \quad \dot{\psi}(0)=0 .
$$

2) Case 2: $\phi(0) \neq 0$ : In this case, the rotor initial velocity needs to be solved for using (10). Note that if we are starting from rest, the initial scaled momentum can be set to zero $\rho(0)=0$. After substituting for $\delta$ from (23) we arrive at

$$
\psi(0)=0, \quad \dot{\psi}(0)=\lim _{t \rightarrow 0} \frac{L^{2} M \tilde{u}}{\tilde{v}^{2} J_{r}}+\frac{M \tilde{v}^{4}}{\tilde{u} J_{r}} .
$$

\section{Solution when $\tilde{v}=0$}

The solution computed above assumes that $\tilde{v}(t) \neq 0$. However, if $\tilde{v}(t)=0$ at some or all points of the trajectories, a gait can still be computed. Indeed, when both $\tilde{v}(t)=\dot{\tilde{\theta}}=0$ a solution is $\tilde{\delta}=0$ with $\tilde{\phi}$ arbitrary, and when $\dot{\tilde{\theta}} \neq 0$ a solution is $\tilde{\phi}= \pm \pi / 2$ with $\tilde{\delta}= \pm M L^{2} \dot{\tilde{\phi}}$. The trajectory can be amended with the solution to these special cases if necessary.

Intuitively, the gait generated for a trajectory where $\tilde{v}=0$ requires either that the Snakeboard is not moving or that the Snakeboard is turning in place around its center of mass.

\section{EXAMPLES}

In this section, we work out some examples and compute the gaits that generate the desired trajectories. We use the following Snakeboard parameters, $M=4, J_{r}=2, J_{w}=\frac{1}{2}$, $J=1$, and $L=1$ in all examples. In each case we specify the 
desired trajectory along which the Snakeboard is supposed to locomote. Then we solve for the respective closed-form gaits.

\section{A. Serpenoid curve}

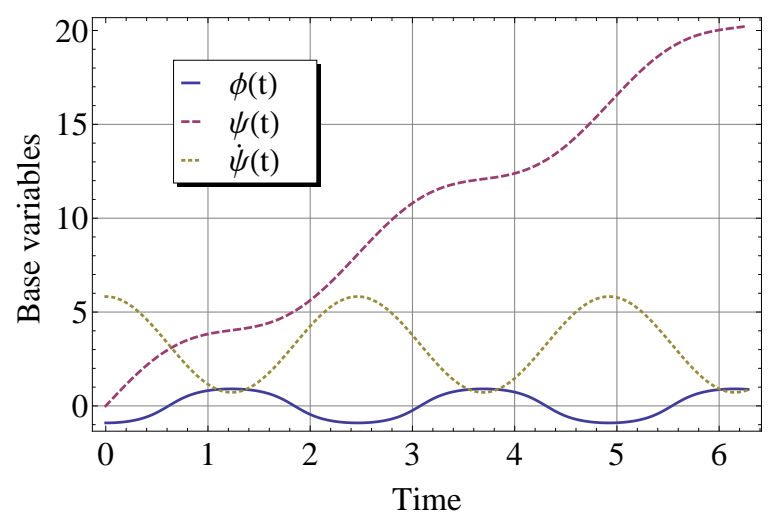

Fig. 3. The Snakeboard gait that was generated to traverse the Serpenoid trajectory depicted in 27.

The Serpenoid curve was first introduced by Hirose [3] to describe snake locomotion. It can be defined as follows

$$
\dot{x}=\cos (a \sin (b t)), \quad \dot{y}=-\sin (a \sin (b t)),
$$

where $a$ and $b$ are parameters of the Serpenoid curve. A parametric plot of the desired Serponoid curve is depicted as a dashed line in Figure 4. Using equation (21) we solve for the wheel direction angle $\phi$ as shown in (28). Then after verifying that $\phi(0) \neq 0$ we use (24) and (26) to solve for the rotor angle as shown (29). The base variables and the first derivative of the rotor angle are depicted in Figure 3.

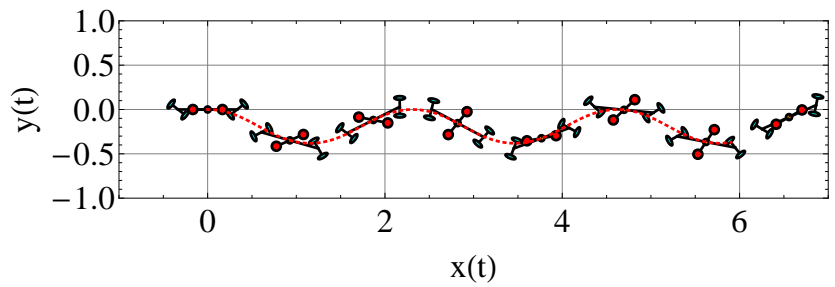

Fig. 4. The Snakeboard snapshots from the numerical simulation of the gaits in 28 and 29 match the parametric plot of the desired trajectory in 27.

$$
\begin{aligned}
\phi & =-\tan ^{-1}(a b L \cos (b t)) \\
\psi & =\frac{M}{a b J_{r}}\left(t+a^{2} b L^{2} \sin (b t)\right)
\end{aligned}
$$

Finally, we numerically simulate the generated gaits depicted in (28) and (29). Snapshots of the Snakeboard locomotion are plotted on top of the parametric plot of the desired trajectory in Figure 4 The simulation shows an exact match between the Snakeboard locomotion and the desired trajectory.

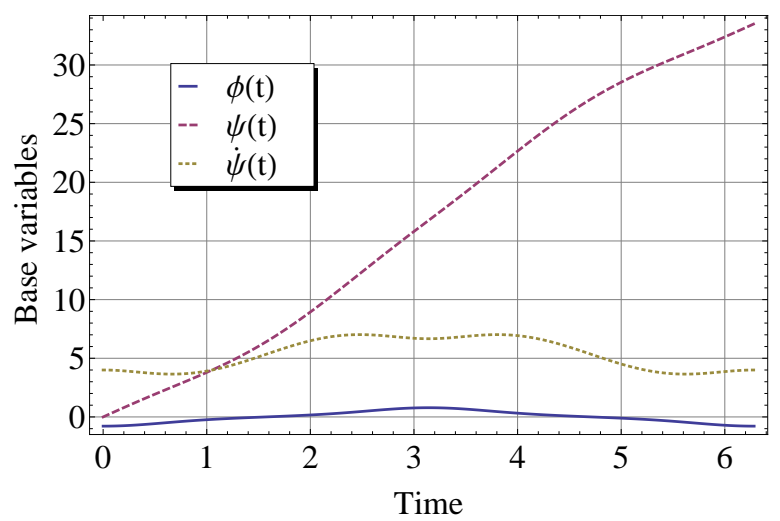

Fig. 5. The Snakeboard gait that was generated to traverse the sinusoidal trajectory depicted in 30 .

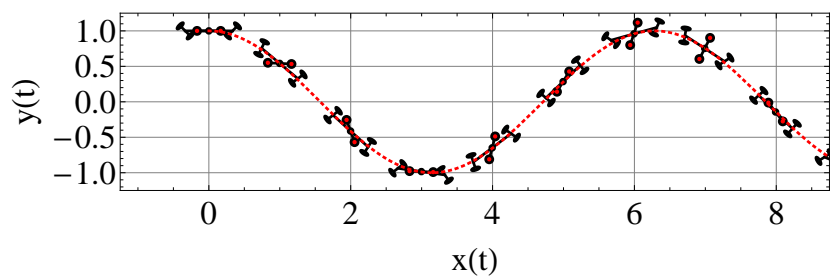

Fig. 6. The Snakeboard snapshots from the numerical simulation of the gaits in 31 and 32 matches the parametric plot of the desired trajectory in 30 .

\section{B. Sinusoidal trajectory with $\phi(0) \neq 0$}

In this example, we want the Snakeboard to follow the following sinusoidal trajectory,

$$
x=t, \quad y=\cos (t) .
$$

A parametric plot of the trajectory is depicted in Figure 5 Again we use equation (21) to solve for the wheel direction angle $\phi$. Then after verifying that $\phi(0) \neq 0$ we use (24) and (26) to solve for the rotor angle to arrive at

$$
\begin{aligned}
\phi= & -\tan ^{-1}\left(\frac{L \cos (t)}{\left(\sin (t)^{2}+1\right)^{\frac{3}{2}}}\right), \\
\psi= & \frac{M}{J_{r}}\left(\frac{8 t}{3}+L^{2} \tan ^{-1}(\sin (t))\right) \\
& +\frac{M}{J_{r}}\left(\frac{\sin (3 t)}{36}-\frac{7 \sin (t)}{4}\right) .
\end{aligned}
$$

The base variables and the first derivative of the rotor angle are depicted in Figure 5] In this example, numerical simulation of the generated gait locomotes the Snakebord along the desired trajectory are shown in Figure 6

\section{Sinusoidal trajectory with $\phi(0)=0$}

Now, we test our gait generation technique for a desired trajectory where the initial wheel angle starts at zero. To demonstrate this, another sinusoidal trajectory is proposed such that

$$
x=t, \quad y=\sin (t) .
$$




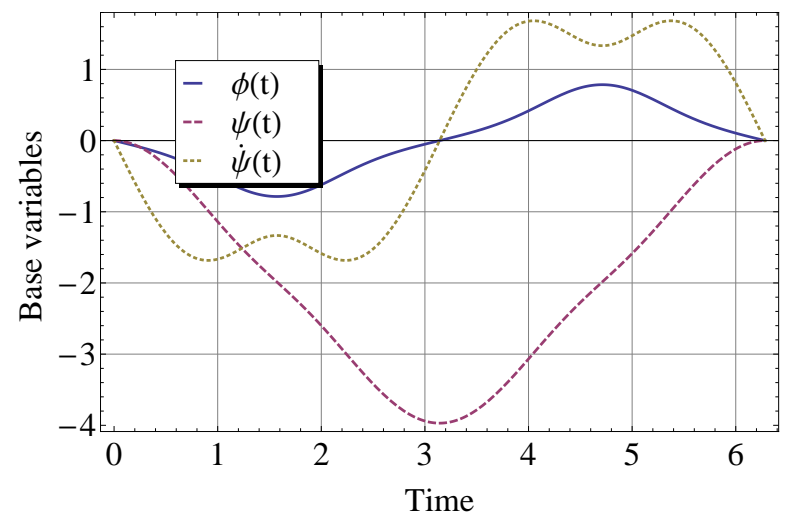

Fig. 7. The Snakeboard gait that was generated to traverse the sinusoidal trajectory depicted in 33 .

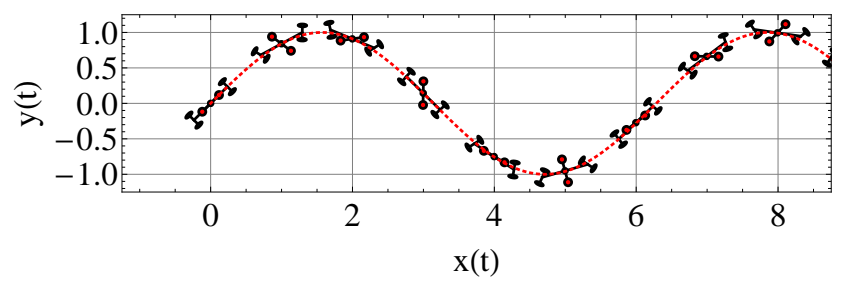

Fig. 8. The Snakeboard snapshots from the numerical simulation of the gaits in 34 and 35 matches the parametric plot of the desired trajectory in 33 .

Indeed for this desired trajectory, after solving for wheel direction angle $\phi$ using equation 21) we arrive at $\phi(0)=0$. Hence, we use the initial conditions given in (25), that is, $\dot{\psi}(0)=0$. Solving for the rotor angle we get

$$
\begin{aligned}
\phi= & -\tan ^{-1}\left(\frac{L \sin (t)}{\left(\cos (t)^{2}+1\right)^{3 / 2}}\right) \\
\psi= & \frac{M}{J_{r}}\left(\frac{\pi L^{2}}{4}-\frac{16}{9}-L^{2} \tan ^{-1}(\cos (t))\right) \\
& +\frac{M}{J_{r}}\left(\frac{\cos (3 t)}{36}+\frac{7 \cos (t)}{4}\right) .
\end{aligned}
$$

For such trajectories, the initial velocity can be arbitrary. In fact, if a different initial rotor velocity was used, the Snakeboard would still traverse the desired trajectory depicted in Figure 8. However, the momentum and scaled momentum variables would change between the two different initial conditions. Moreover, the $\delta$ momentum variable is not affected by the initial rotor velocity condition. To demonstrate this result, gaits were generated for the initial condition $\dot{\psi}(0)=1$ rather than $\dot{\psi}(0)=0$. The momentum variable time evolution for both cases are shown in Figure 9

\section{Cubic spline trajectory}

In this last example, we want to design a gait that drives the Snakeboard from a start to a final position. To completely define the trajectory that connects the start and end positions, we specify the slope of the trajectory at these position. Hence, a cubic spline can be used to define such a trajectory. Without any loss of generality, we specify the trajectory to start from

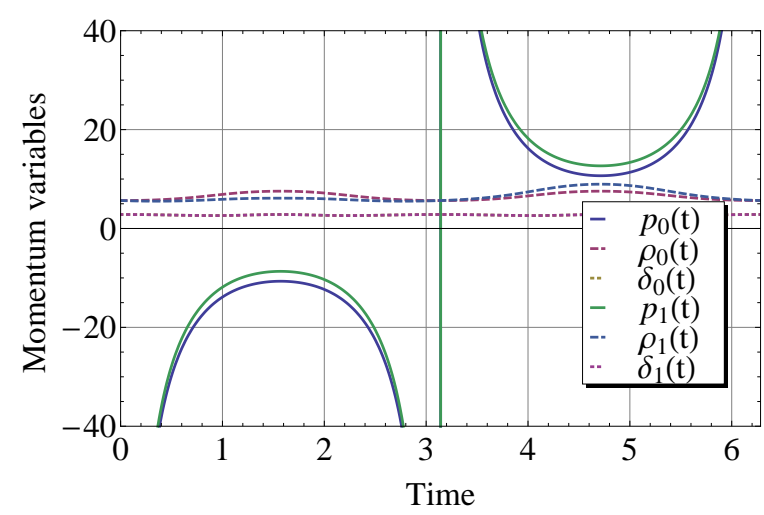

Fig. 9. A plot of the momentum variable for the two initial rotor velocity conditions. $\left(p_{0}(t), \rho_{0}(t), \delta_{0}(t)\right)$ correspond to $\dot{\psi}(0)=0$ while $\left(p_{1}(t), \rho_{1}(t), \delta_{1}(t)\right)$ correspond to $\dot{\psi}(0)=1$.

the origin with the middle link horizontal and to end at the point $\left(1, y_{f}\right)$ with the middle link having a slope $m$. Such a trajectory can be computed as follows

$$
\begin{aligned}
& x=t, \quad y=a t^{2}+b t^{3}, \quad \text { where } \\
& a=3 y_{f}-m, \text { and } b=m-2 y_{f} .
\end{aligned}
$$

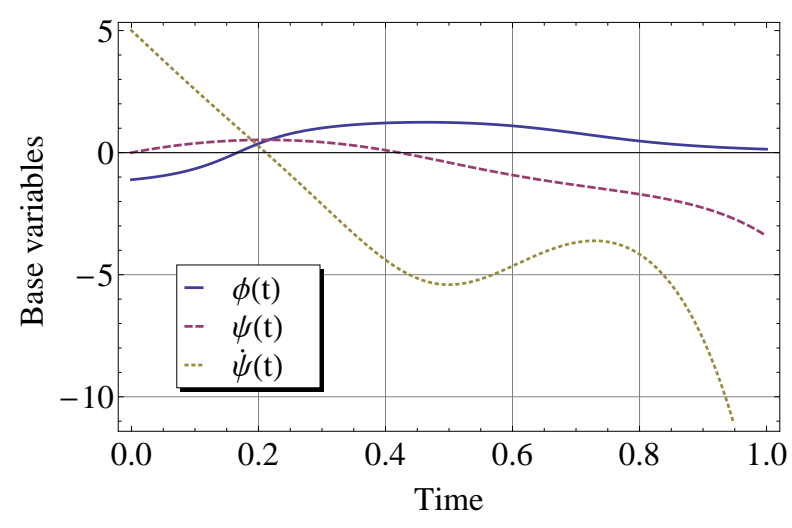

Fig. 10. The Snakeboard gait that was generated to traverse the cubic spline trajectory depicted in 36 .

The above trajectory is depicted in Figure 10 . Using equation (21) we solve for the wheel direction angle $\phi$. Then after verifying that $\phi(0) \neq 0$ we use (24) and (26) to solve for the rotor angle to arrive at

$$
\begin{aligned}
\phi= & \tan ^{-1}\left(\frac{2 L(a+3 b t)}{\left(\left(2 a t+3 b t^{2}\right)^{2}+1\right)^{3 / 2}}\right), \\
\psi= & -\frac{2 a^{3} M t^{5}}{5 J_{r}}-\frac{6 a^{2} b M t^{6}}{5 J_{r}}-\frac{9 a b^{2} M t^{7}}{7 J_{r}} \\
& -\frac{L^{2} M \tan ^{-1}(t(2 a+3 b t))}{a M t^{3}}-\frac{M t}{3 J_{r}}-\frac{J_{r}}{2 a J_{r}} \\
& -\frac{27 b^{3} M t^{8}}{56 J_{r}}-\frac{b M t^{4}}{4 J_{r}} .
\end{aligned}
$$




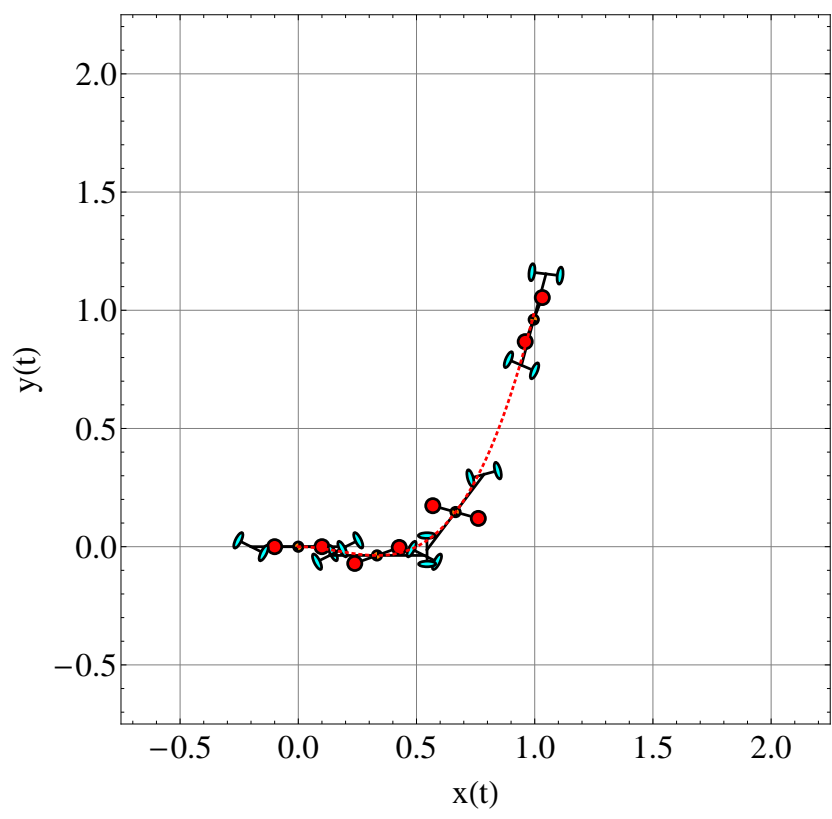

Fig. 11. The Snakeboard snapshots from the numerical simulation of the gaits in 38 and 39 matches the parametric plot of the desired trajectory in 36 .

After numerically simulating the generated gaits, the Snakeboard exactly traverses the desired trajectory as shown in Figure 11.

In summary, in this section, we have generated four sets of gaits by analytically solving for the base variables $\phi$ and $\psi$ for several desired trajectories. In all the examples, the generated gaits drove the Snakeboard exactly along the respective desired trajectories.

\section{Discussion}

Now we address some assumptions that were enforced earlier and verify that the gait generation techniques are not affected by these assumptions; Moreover, some intuition is given for the new momentum-like variable introduced in this paper.

\section{A. Snakeboard simplification}

In this paper we introduced two main simplification to the Snakeboard, namely, coupling the front and back wheel directions and the condition that we used on the total inertia of the Snakeboard, $M L^{2}=J+J_{r}+2 J_{w}$. Similar to all prior literature relating to the Snakeboard we maintain the coupling of the wheel direction; However, removing the inertia simplification does not affect the gait generation technique that was presented in this paper. In fact, removing the inertia relation and letting $J=m L^{2}-J_{r}-2 J_{w}$ we get

$$
\begin{aligned}
\theta & =\tan ^{-1} \frac{\dot{\tilde{y}}}{\dot{\tilde{x}}}, \\
\phi & =-\tan ^{-1}\left(\frac{L \tilde{u}}{\tilde{v}^{3}}\right), \\
\delta & =-\frac{L \sqrt{-L^{2} m \tilde{u}^{2}-M \tilde{v}^{6}}}{\sqrt{2} \tilde{v}^{2}}, \\
\ddot{\psi} & =\frac{L^{2} m \dot{\tilde{u}}}{J_{r} \tilde{v}^{2}}-\frac{2 L^{2} m \tilde{u} \dot{\tilde{v}}}{J_{r} \tilde{v}^{3}}+\frac{M \tilde{v}^{3} \dot{\tilde{v}}}{J_{r} \tilde{u}}, \text { and } \\
\dot{\psi}(0) & =\lim _{t \rightarrow 0} \frac{L^{2} m \tilde{u}}{J_{r} \tilde{v}^{2}}+\frac{M \tilde{v}^{4}}{J_{r} \tilde{u}},
\end{aligned}
$$

where $\tilde{u}$ and $\tilde{v}$ are still as defined in 201. Note that both $\theta$ and $\phi$ were not affected by the inertia simplification; However, the expressions of $\delta$, and $\ddot{\psi}$ and its initial velocity, $\dot{\psi}(0)$ have changed. Nevertheless, the method of generating gaits presented in Section III is still valid.

\section{B. Momentum variables}

This paper introduces yet another momentum-like variable to achieve the explicit gait generation techniques. The nonholonomic momentum variable as described in Ostrowski [7], $p(t)$ is computed along the allowable directions of motion and defined by

$$
p(t)=\frac{\partial l(\xi, r, \dot{r})}{\partial \xi} \Omega(r),
$$

where $l(\xi, r, \dot{r})$ is the reduced Lagrangian and $\Omega(r)$ ia a basis of the nullspace of the matrix $\omega_{\xi}(r)$ which in turn is a sub-matrix of the reduce nonholonomic constraints matrix satisfying $\omega_{\xi}(r) \xi+\omega_{r}(r) \dot{r}=0$.

The second momentum variable discussed in this paper is the scaled momentum given by $\rho(t)=f(r(t)) p(t)$ as was defined in Shammas et al. [8]. The scaled momentum is simply the nonholonomic momentum, $p$ multiplied by an integrating factor, $f(r)$, to simplify the momentum evolution equation.

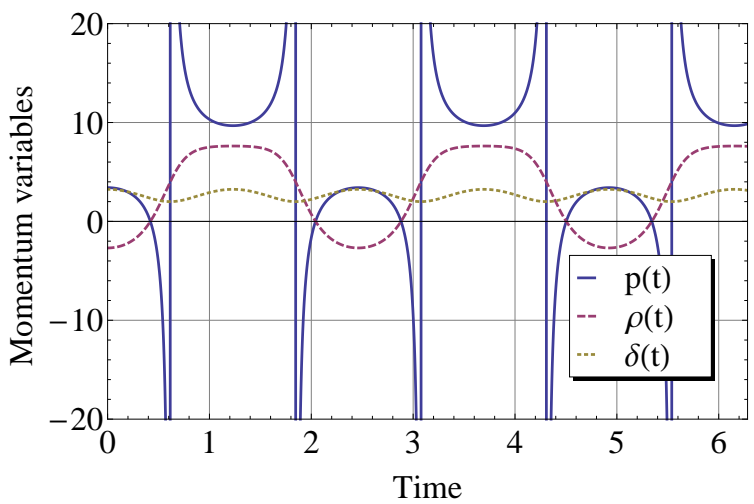

Fig. 12. A plot of the different momentum variables for the Seprenpoid trajectory shown in Figure 4

Finally, we introduces a third momentum related variable, $\delta$, that greatly reduces the nonholonomic equations of motion 
and that is crucial to the explicit gait generation technique in this paper. Pertaining to the Snakeboard, there are two interesting facts regarding this momentum variable. First, its first derivative is a function of the base variables, $r$, and their second time derivatives, $\ddot{r}$ as shown in 47); Moreover, the right-hand-side of the momentum evolution equation is not a quadratic-form. The second interesting fact is that the reconstruction equation when written in term of the new momentum variable, $\delta$, its right-hand-side is not dependent on the base velocities, $\dot{r}$, as shown in (46). Thus we can conclude that the reconstruction and momentum evolution equation for the Snakeboard when expressed in terms of $\delta$ are given by

$$
\begin{aligned}
& \xi=\frac{1}{f(r)} \Gamma(r) \delta \\
& \dot{\delta}=h(r) \ddot{r}
\end{aligned}
$$

where $h(r)$ is a function of the base variable configurations. The difference between the three momentum variables discussed in this paper is depicted in Figure 12

\section{Allowable trajectories}

In this paper we solved for gaits that locomote the Snakeboard along planar trajectories comprised of what we labeled as "sufficiently smooth" curves. To be more precise, the components of the parametric representation of the trajectory must be twice differentiable with respect to time and the second derivatives must be continuous. These conditions were sufficient to solve for the gaits for all the example trajectories presented in the previous section.

If a desired trajectory is represented by a cubic spline or is composed of several curves from the families of curves from the previous section, the gait generation methods in the paper are still valid as long as there are no kinks in the second derivatives of the components of the curve. In other words, when constructing a feasible trajectory for the Snakeboard to traverse, one should ensure that the third derivative of the components of the desired trajectory are continuous and the derivatives up to second order are identical at the intersection between two adjacent trajectory section.

Finally, it is worth noting that equation (22) imposes no limits on the radius of curvature along the desired trajectory. Indeed, when $\phi= \pm \pi / 2$, the radius of curvature is zero, that is, the wheel axes are along the middle link of the Snakeboard and it turns in place.

\section{CONCLUSION}

In this paper we presented a closed-form analytical solution to the motion planning problem of the Snakeboard, that is, given any sufficiently smooth curve in the plane, respective base variables can be solved for so that the Snakeboard exactly traverses the desired trajectory. This result was achieved by introducing a momentum-like variable that simplified the reduced nonholonomic equations of motion so that an explicit inverse dynamics solutions is feasible.

Generalizations of this technique to a subclass of simple mechanical systems will be investigated in the near future.
More importantly, the existence of the new momentum-like variable and the form of its evolution differential equation should be investigated for other mechanical systems.

Finally, it would be interesting to parameterize the desired trajectories in terms of curvature as a function of arc-length. Such a parametrization could significantly simplify the inverse dynamics problem as well as provide some intuition for the nature of the new momentum-like variable.

\section{REFERENCES}

[1] F. Bullo and A.D. Lewis. Kinematic controllability and motion planning for the snakeboard. Robotics and Automation, IEEE Transactions on, 19(3):494 - 498, 2003.

[2] F. Bullo and K.M. Lynch. Kinematic controllability for decoupled trajectory planning in underactuated mechanical systems. Robotics and Automation, IEEE Transactions on, 17(4):402 -412, August 2001.

[3] Shigeo Hirose. Biologically Inspired Robots: Serpentile Locomotors and Manipulators. Oxford University Press, 1993.

[4] R.M. Murray and S.S. Sastry. Nonholonomic motion planning: steering using sinusoids. Automatic Control, IEEE Transactions on, 38(5):700 -716, May 1993.

[5] J. Ostrowski, A. Lewis, R. Murray, and J. Burdick. Nonholonomic mechanics and locomotion: the snakeboard example. In Robotics and Automation, 1994. Proceedings., 1994 IEEE International Conference on, pages 2391 -2397 vol.3, May 1994.

[6] J. Ostrowski, J.P. Desai, and V. Kumar. Optimal gait selection for nonholonomic locomotion systems. In Robotics and Automation, 1997. Proceedings., 1997 IEEE International Conference on, volume 1, pages 786 -791 vol.1, April 1997.

[7] James Patrick Ostrowski. The Mechanics and Control of Undulatory Robotic Locomotion”. PhD thesis, California Institute of Technology, Pasadena, CA 91125, 1995.

[8] E. Shammas, H. Choset, and A. Rizzi. Towards automated gait generation for dynamic systems with nonholonomic constraints. In Robotics and Automation, 2006. ICRA 2006. Proceedings 2006 IEEE International Conference on, pages 1630 -1636, May 2006.

[9] Elie A. Shammas, Howie Choset, and Alfred A. Rizzi. Geometric motion planning analysis for two classes of underactuated mechanical systems. The International Journal of Robotics Research, 26(10):1043-1073, 2007.

[10] Elie A. Shammas, Howie Choset, and Alfred A. Rizzi. Towards a Unified Approach to Motion Planning for Dynamic Underactuated Mechanical Systems with Nonholonomic Constraints. The International Journal of Robotics Research, 26(10):1075-1124, 2007. 